\title{
Analysis of the Index Test method of Optical Terminal in Optical Fiber Communication
}

\author{
Wu Xinmin ${ }^{1, a}$ \\ 1. ZheJiang University of Water Resources and Electric Power, Hangzhou 310000, ZheJiang , \\ China \\ awuxm@zjweu.edu.cn
}

Keywords: optical transceiver; the average optical power; extinction ratio; sensitivity; dynamic range; testing method

Abstract. the optical transmitter and optical receiver composed by light. Two important indicators of the optical transmitter average optical power, extinction ratio of two important indicators and optical receiver sensitivity, dynamic range are analyzed, and the importance of their carefully introduced test method. They not only directly for the communication service, and the theoretical calculations are correct for the test, and then modify and improve the design scheme. Therefore, this analysis for everyone in the experiment, the construction and maintenance work has its important significance on four index and test method.

\section{Introduction}

With the rapid development of modern communication technology, optical fiber communication technology has become an important means of communication ${ }^{[1]}$. In the operation of optical fiber transmission system, the average optical power, extinction ratio of optical terminal, The receiving sensitivity and dynamic range of four parameters are very important.

\section{Testing of optical transmitter}

The optical transmitter consists of an input code type conversion circuit and an optical transmission circuit ${ }^{[2]}$. The optical transmitter converts the digital signal output from the electric terminal to the light source by direct light intensity modulation into an optical signal. The coupling technique is used to effectively inject the fiber line. The test principle is shown in figure 1.

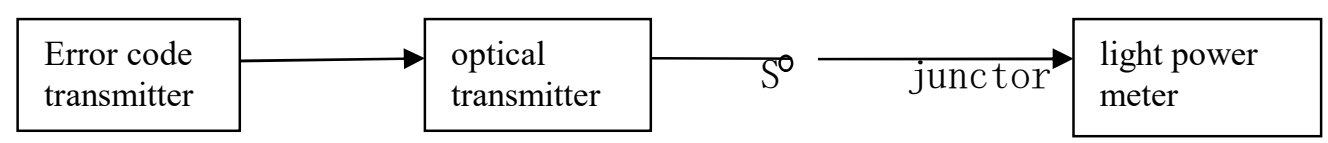

Fig. 1 Test schematic diagram of optical transmitter

\section{Measurement of average optical power}

In the testing of optical fiber communication, the test of many important parameters is actually the test of optical power. The average transmitting optical power of optical terminal is the average optical power output of optical terminal under normal working conditions, that is, the output of light source tail fiber. The average optical power produced. The index of the average optical power is related to the actual optical fiber line. In the long distance optical fiber communication digital system, the average transmitting optical power is required, while in the short distance optical fiber communication system, A smaller average transmitted optical power is required ${ }^{[3]}$. The test method of average optical power is as follows: $2^{23}-1$ pseudorandom code or $2^{15}-1$ pseudorandom code is transmitted from the error code meter, and the light source is connected with the optical power meter to output the tail fiber connector. The output average optical power of the transmitter can be measured. However, it should be noted that the value of the average optical power is related to the selected code type, such as the RZ 
code with the NRZ code ratio $50 \%$ duty cycle, the power is larger than $3 \mathrm{~dB}$, and the choice of the power meter should be paid attention to in the measurement. Short wavelength optical power meter must be used in short wavelength system, long wavelength optical power meter must be used in long wavelength system, and optical power is usually expressed by "dBm" or "UW".

The steps for measuring the average optical power are as follows:

(1) Connect the circuit according to the test block diagram.

(2) Remove the fiber plug from the receiver connector and connect with the optical power meter.

(3) Optical terminals send PCM test signals, and optical fiber digital communication systems with different code speeds require different PCM test signals.

(4) Turn on the power switch of the error analyzer, and set the speed of the error analyzer to 2048 $\mathrm{kb} / \mathrm{s}$, the pattern to $2^{15}-1$, and the code type to NRZ.

(5) Read the optical power value from the optical power meter, that is, the average transmitted optical power, and record it.

\section{Measurement of extinction ratio}

Extinction ratio (EX) is one of the quality indexes of the transmission part of the optical terminal. It is defined as the ratio of the average power when the light source emits the full " 1 " and the " 0 " codes. Since the general code type generator sends out pseudo-random codes, it can be considered that the occurrence probability of the " 1 " code " 0 " codes is equal. Therefore, the optical power at full " 1 " code is equal to twice the average optical power, so the extinction ratio can be expressed as:

$$
\mathrm{EX}=10 \lg \frac{2 P_{a}}{P_{0}}
$$

Of which: $\mathrm{P}_{0}$ Output optical power for full " 0 " code, $\mathrm{Pa}$ is the average optical power of output pseudorandom code

The test schematic diagram of extinction ratio is shown in figure 1 . The test method is that the error code instrument sends out $2^{23}-1$ or $2^{15}-1$ pseudorandom code and measures the average optical power at this time $\mathrm{Pa}$. The input point in the optical transmitter is grounded and the total "0" code optical power $\mathrm{P}_{0}$. the extinction ratio can be obtained by calculating the formula of " 0 " code optical power.

Test steps for extinction ratio:

(1) Connect the circuit according to the test block diagram.

(2) Send the required test signal.

(3) The optical power measured when a full " 0 " code is input is when the input signal is cut off (usually the encoding disk is pulled out)

(4) Read out the optical power from the optical power meter , And take notes.

(5) The average transmitted optical power to be obtained and The extinction 1-1ratio is obtained.

\section{Optical receiver testing}

The optical receiver is composed of an optical receiving amplifier circuit and an input code type conversion circuit. The purpose of the receiver is to convert the weak optical signal whose amplitude is attenuated after the light is transmitted and the waveform is broadened to an electric signal, and to process the amplification. Reverts to the original transmitted digital sequence. The test principle is shown in figure 2 .

\section{Sensitivity test}

The sensitivity of the optical receiver is defined as the minimum power to be received at a certain bit error rate, which represents the ability of the optical receiver to receive weak signals. The test frame is shown in figure 2. Receiver sensitivity is the minimum average optical power that an optical terminal can receive under a given bit error rate or signal-to-noise ratio. Sensitivity is an important 
performance index of an optical terminal, which indicates that an optical terminal receives a weak signal. Therefore, the relaying distance of the system is an important basis for system design ${ }^{[2]}$. When testing the sensitivity of optical receiver, we must first determine the BER index required by the system. For different optical fiber digital communication systems of different lengths and applications, the BER index is different. The lower the bit error rate is, the lower the sensitivity is, that is, the greater the optical power is required. In addition, the sensitivity is also related to the code speed of the system and the type of photo detect or at the receiving end. The test methods are as follows: the sending part of the error meter sends out $2^{23}-1$ or $2^{15}-1$ pseudorandom code, and monitors the error code at the same time, and increases the attenuation of the optical attenuator. Until the bit error rate indicated by the error-meter is tested in optical fiber communication, the test of many important parameters is actually a test of optical power. The minimum optical power Pmin, that is, the sensitivity of the optical receiver. The expression of sensitivity is generally expressed by $\mathrm{dBm}$ :

$$
\operatorname{Pr}=10 \lg \frac{P \min }{1 m W} \quad(\mathrm{dBm})
$$

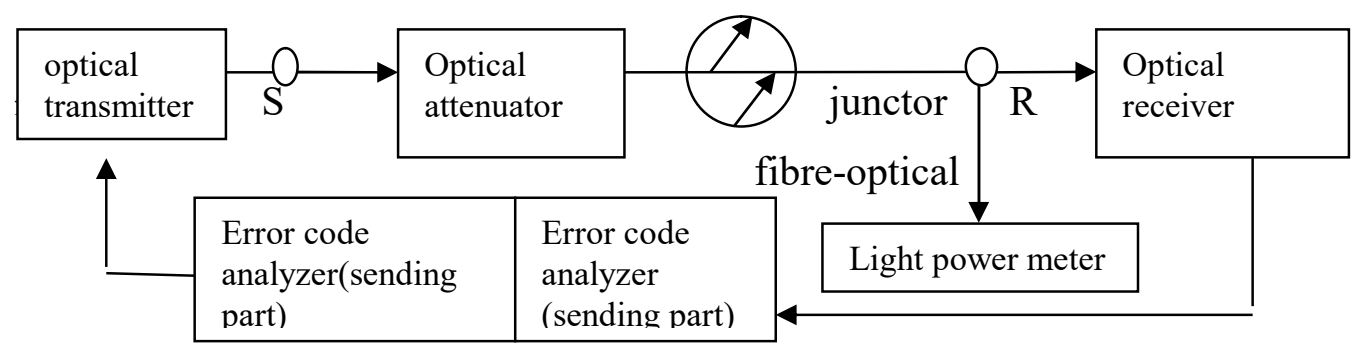

Fig. 2 testing principle diagram of optical receiver

It should be noted that because the sensitivity measurement is measured before the connector (including a live connector), the actual sensitivity should be subtracted from the loss (or description) of the connector; in order to facilitate the adjustment of the received optical power, In the test, the optical attenuator is used instead of the long fiber, so the influence of fiber dispersion on the sensitivity is ignored, so in practical use, The influence of dispersion on the sensitivity should be estimated according to the length and quality of the fiber, or measured directly in the circuit with long optical fiber. In addition, the weak output power should be measured by using a light detector and an optical power meter with a chopper.

Sensitivity test steps:

(1) Connect the line according to the test block diagram.

(2) The test signal is sent from the error detector to the optical end, and the selection of different signals and the optical power test are the same.

(3) The attenuation of the optical variable attenuator is adjusted so that the optical power is at a certain value (such as $23 \mu \mathrm{W}$ or so), the optical fiber is inserted into the optical receiving module, and the bit error rate is measured and recorded.

(4) If the BER is $10^{-3}$, then the optical power is the minimum power Pmin.If not $10^{-3}$, repeat step $3 / 4$ until the BER is $10^{-3}$.

(5) According to the test results, the sensitivity of the optical receiver is calculated by substitution.

\section{Dynamic range testing}

The dynamic range of the optical receiver is defined as the ratio of the maximum to the minimum input optical power allowed under certain bit error rates. It represents the adaptability of the optical receiver to the variation of the input signal ${ }^{[4]}$. The test block diagram is shown in figure 2 . The measuring method is basically the same as the method of measuring sensitivity, but only the maximum input optical power is added to the measurement. The method is to gradually reduce the 
attenuation of the optical attenuator. The received optical power is the maximum input optical power Pmax the BER is reduced to a required value (e.g.: $10^{-9}$ ).

The dynamic range is represented as:

$$
D r=10 \lg \frac{P \max }{P \min }(\mathrm{dB})
$$

The test method is to connect the line according to the block diagram and send it into the required test signal. Reduce the attenuation of the variable attenuator, make the received optical power increase gradually, after the error code appears, increase the light attenuation, until the bit error rate is just back to the. After the fixed value is fixed and stabilized for a certain time, the power read by the optical power meter is Pmax. the attenuation continues to increase until the critical state of large error code appears and the optical power measured is Pmin.

It is important to note that the test of dynamic range Dr should also take into account the length of test time, and only if the system is within the index of error code requirement for a long time is the power value correct.

The test steps are as follows:

(1) Connect the line according to the test block diagram and send the required test signal.

(2) Adjust the attenuation of the optical variable attenuator so that the optical power is at a certain value (such as $51 \mu \mathrm{W}$ or so), the optical fiber is inserted into the optical receiving module, and the bit error rate is measured and recorded.

(3) If the BER is $10^{-9}$, then the optical power is the maximum optical power Pmax. if not $10^{-9}$, repeat step 2、3 until the BER is $10^{-9}$.

(4) According to the test results, the dynamic range of the optical receiver is calculated in the formula 2-1.

To calculate the dynamic range according to the formula Dr. It is important to note that the test time should also be taken into account in the dynamic range testing, and the power value can be correct only if the system is within the error code requirement index for a long time.

\section{Conclusions}

With the rapid development of artificial intelligence network, industrial Internet, Broadband speed increase, material connection, energy saving and emission reduction all focus on the development of optical fiber broadband access network, and also provide good development opportunities for optical transmission equipment and optical cable manufacturing enterprises, and at the same time expand the scale of the market or upgrade the system products. The optical terminal has a better opportunity. Parameter testing is an important technical index in optical fiber testing. It is very important to the quality of optical fiber. The problems and solutions to the application of test method and the higher requirement of technical index in practical test are summarized.

\section{References}

[1] Ponce Wei. Development and application of optical terminal technology-technology and application [J],2013, 5: p.40 45

[2] Qiao Guihong, Wu Fengxiu, Chen Yiping. fibre communication [M]. Beijing people's post and Telecommunications Press(,2009.7).

[3] Zhang Baofu, Su yang, Wang Haitong. Beijing people's post and Telecommunications Press [M]. Xi'anUniversity of Electronic Science and Technology of XiAn(2017.7).

[4] Zhang chaochun. High definition Video Fiber Transmission system [D]. Chengtu university of science and engineering(2012). 